Draft VERsion June 5, 2021

Preprint typeset using $\mathrm{LAT}_{\mathrm{E}} \mathrm{X}$ style emulateapj v. 5/2/11

\title{
HST/ACS DIRECT AGES OF THE DWARF ELLIPTICAL GALAXIES NGC 147 AND NGC 185
}

\author{
M. Geha ${ }^{1}$, D. Weisz ${ }^{2,3}$, A. Grocholski ${ }^{4}$, A. Dolphin $^{5}$, R. P. van der Marel ${ }^{6}$, and P. Guhathakurta ${ }^{7}$ \\ Draft version June 5, 2021
}

\begin{abstract}
We present the deepest optical photometry for any dwarf elliptical (dE) galaxy based on Hubble Space Telescope ACS observations of the Local Group dE galaxies NGC 147 and NGC 185. The resulting F606W and F814W color-magnitude diagrams are the first to reach below the main sequence turnoff in a $\mathrm{dE}$ galaxy, allowing us to determine full star formation histories in these systems. The ACS fields are located $\sim 1.5$ effective radii from the galaxy center to avoid photometric crowding. While our ACS pointings in both dEs show unambiguous evidence for old and intermediate age stars, the mean age in NGC 147 is $~ 4$ Gyr younger as compared to NGC 185. In NGC 147, only $40 \%$ of stars were in place 12.5 Gyr ago $(\mathrm{z} \sim 5)$, with the bulk of the remaining stellar population forming between 5 to 7 Gyr. In contrast, $70 \%$ of stars were formed in NGC 185 field more than 12.5 Gyr ago, with the majority of the remaining population forming between 8 to 10 Gyr. Star formation ceased in our ACS fields in both dEs at least 3 Gyr ago. Previous observations in the central regions of NGC 185 show evidence for star formation as recent as $100 \mathrm{Myr}$ ago and a strong metallicity gradient with radius. We suggest that the orbit of NGC 185 has a larger pericenter as compared to NGC 147, allowing it to preserve radial gradients and maintain a small central reservoir of recycled gas. We interpret the inferred differences in star formation histories to imply an earlier infall time into the M31 environment for NGC 185 as compared to NGC 147.

Subject headings: galaxies: dwarf — galaxies: stellar content — galaxies: individual (NGC 147, NGC 185)
\end{abstract}

\section{INTRODUCTION}

Dwarf elliptical (dE) galaxies are primarily, if not exclusively, found in galaxy cluster and group environments (Binggeli et al. 1988; Ferguson \& Sandage 1991; Geha et al. 2012). Environment-driven processes must play an important role in $\mathrm{dE}$ formation and evolution (e.g., Moore et al. 1998; Mastropietro et al. 2005; Maver 2010; Lisker et al. 2013). However, no single process or formation mechanism can yet explain the observed diversity of $\mathrm{dE}$ morphologies (e.g., Lisker et al. 2009; Janz et al. 2012) or kinematics (e.g., Geha et al. 2002; van Zee et al. 2004; Toloba et al. 2014). Star formation histories are an important tool in understanding the $\mathrm{dE}$ galaxy class and provide insight into the assembly of galaxies in dense environments.

Stellar population studies in the nearby Virgo and Fornax galaxy clusters reveal a mixture of old to intermediate age stars in $\mathrm{dE}$ galaxies, and intrinsic differences in the age and/or metallicity of individual galaxies Geha et al. 2003; Michielsen et al. 2008; Ryś et al. 2013). These studies are based on either integrated spectral line widths or narrow band imaging and cannot therefore determine the exact ratio of old to intermediate

\footnotetext{
${ }^{1}$ Astronomy Department, Yale University, New Haven, CT 06520. marla.geha@yale.edu

2 Astronomy Department, Box 351580, University of Washington, Seattle, WA

${ }^{3}$ Hubble Fellow

${ }^{4}$ Department of Physics \& Astronomy, Louisiana State University, Baton Rouge, LA 70803

${ }^{5}$ Raytheon, 1151 E. Hermans Road, Tucson, AZ 85756, USA

${ }^{6}$ Space Telescope Science Institute, 3700 San Martin Drive, Baltimore, MD 21218

${ }^{7}$ UCO/Lick Observatory, University of California, Santa Cruz, 1156 High Street, Santa Cruz, CA 95064.
}

stars, nor reconstruct detailed star formation histories. The Local Group dEs share the same broad properties as galaxy cluster dEs (Bender et al. 1991), yet are sufficiently nearby that individual stars can be resolved below the main sequence turn-off, allowing for more unambiguous stellar population estimates in these systems.

The Local Group contains three dE galaxies: NGC 205, NGC 147 and NGC 185, all satellites of the large spiral galaxy M31. A fourth bright satellite, M 32, is far more compact and likely has a very different formation path (Monachesi et al. 2012). Of the three dEs, NGC 205 is close in projection to the M31 disk, and contamination from M31 stars complicates stellar population work in this object (Choi et al. 2002). The stellar populations of NGC 147 and NGC 185 are more amenable to study. RR Lyrae stars are detected in both NGC 147 and NGC 185 indicative of stars older than $10 \mathrm{Gyr}$ Saha \& Hoessel 1990; Yang \& Sarajedini 2010). AGB carbon stars are also observed in both dEs, typical of more intermediate age stars (Battinelli \& Demers 2004; Davidge 2005; Sohn et al. 2006). Each of these galaxies hosts its own system of associated globular clusters (Veljanoski et al. 2013).

NGC 147 and NGC 185 share fundamental properties, such as absolute luminosity $\left(M_{V} \sim-16\right.$; Crnoiević et al. 2014) and velocity dispersion $(25 \mathrm{~km} / \mathrm{s}$; Geha et al. 2010). However, they differ in many aspects. NGC 185 contains some gas, dust and evidence for recent star formation confined to its center, while NGC 147 is devoid of gas or dust and shows no sign of recent star formation activity (Young \& Lo 1997; Marleau et al. 2010). HST/WFPC2 images of both galaxies also imply the presence of intermediateage stars, with NGC 147 having a more significant con- 

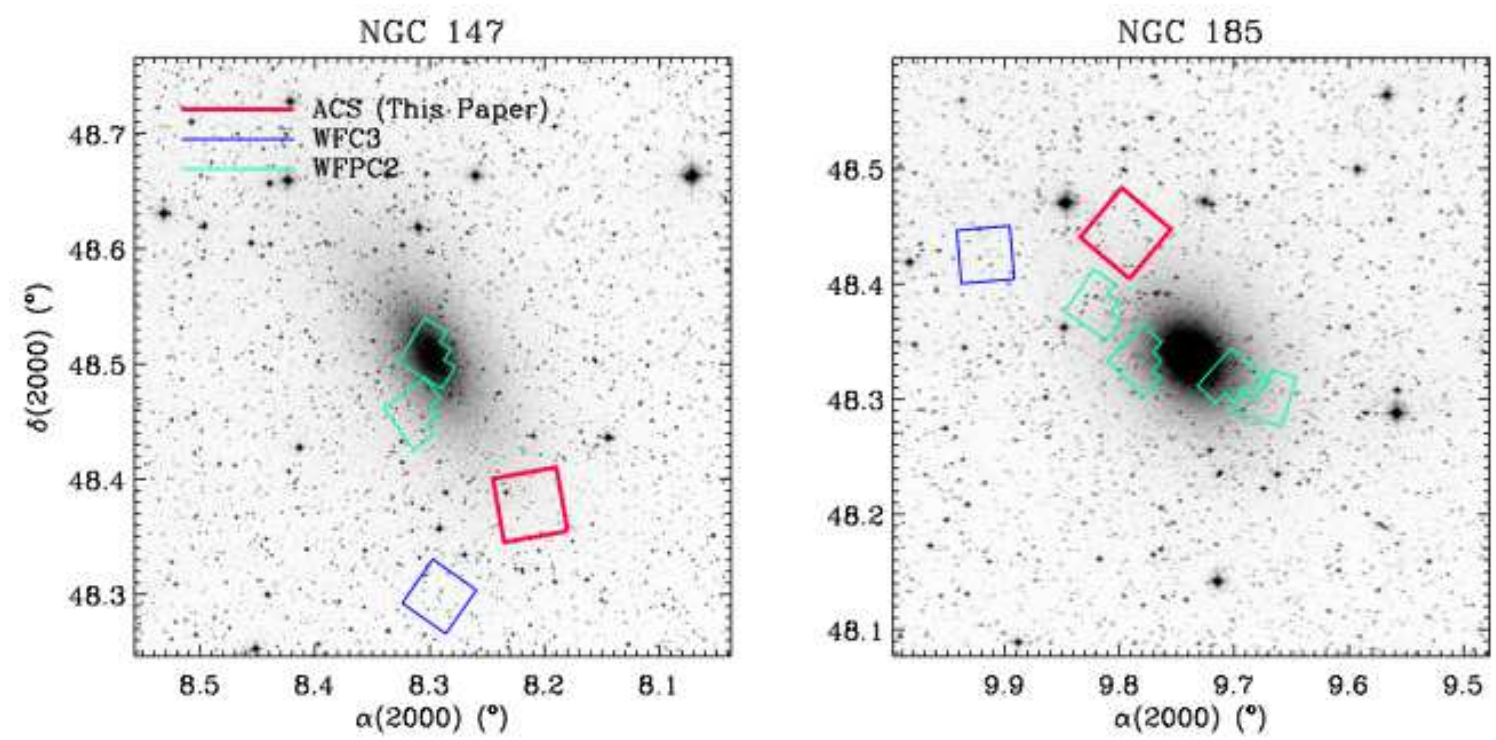

Fig. 1.- Digital Sky Survey images of the Local Group dE galaxies, NGC 147 (left) and NGC 185 (right). The ACS pointings discussed in this paper are shown in red. WFC3 pointings taken in parallel with the ACS data are shown in blue and will be analyzed in a future paper. Archival $H S T /$ WFPC2 pointings are shown in green. Images are $30^{\prime} \times 30^{\prime}$; North is up, East is to the left.

tribution than NGC 185 based on stars brighter than the main sequence (Butler \& Martínez-Delgado 2005; Weisz et al. 2014). Spectroscopic metallicities show an overall more metal-rich population in NGC $147,[\mathrm{Fe} / \mathrm{H}]=$ $-0.5 \pm 0.1$ as compared to NGC 185 of $[\mathrm{Fe} / \mathrm{H}]=-0.9 \pm 0.1$ (Vargas et al. 2014). Only NGC 185 shows evidence for a metallicity gradient, becoming more metal-poor with radius (Vargas et al. 2014; Crnojević et al. 2014

NGC 147 and NGC 185 are very close in projection on the sky $\left(58^{\prime}\right)$, leading some to argue that they may be a bound galaxy pair (van den Bergh 1998). However, kinematic evidence suggests that these may not be gravitationally bound (Geha et al. 2010; Watkins et al. 2013). Furthermore, deep photometry from the Pan-Andromeda Archaeological Survey (PAndAs) has uncovered isophotal twisting at large radius due to the emergence of symmetric tidal tails in NGC 147, which is not seen to similar depths in NGC 185 (Crnojević et al. 2014), further suggesting these two objects have independent formation paths.

In this paper, we present deep Hubble Space Telescope (HST) Advanced Camera for Surveys (ACS) imaging for NGC 147 and NGC 185. These are the only two dE galaxies for which $H S T$ can cleanly resolve stars below the main sequence turnoff region and thus directly measure their star formation histories. The paper is organized as follows: in $\oint 2$ we discuss the HST observations and data reduction. We first compare the resulting colormagnitude diagrams to single stellar population models (\$3.1), and then compute full star formation histories $(\S 3.3)$. In $\S 4$, we discuss our results in the context of $\mathrm{dE}$ galaxy formation.

\section{DATA}

\subsection{Image Reduction and Photometry}

The HST/ACS observations were taken for NGC 147 between 2009 November-December, and for NGC 185 between 2010 January-February (HST GO-11724; PI: Geha). We note that this project was first approved as HST-GO-10794 (PI: Geha), but was not completed un- der this program number after the 2007 ACS failure. Our post-servicing mission ACS fields were chosen to avoid crowding while ensuring a well-populated CMD in the F606W (broad $V$ ) and F814W (I) filters, corresponding to a surface brightness of $\mu_{V} \sim 26 \mathrm{mag} \operatorname{arcsec}^{-2}$. The exact field locations were chosen to avoid bright foreground stars at this radius. Parallel WFC3 observations were taken as part of this program. A quick reduction of the WFC3 fields suggest a much sparser stellar density as expected, but similar stellar populations as the primary ACS fields; these data will be analyzed in a future contribution.

The ACS fields are located roughly along the major axis of each galaxy (Figure 1), at $8.6^{\prime}(1.8 \mathrm{kpc})$ and $6.7^{\prime}$ $(1.2 \mathrm{kpc})$ from the galaxy center for NGC 147 and NGC 185, respectively. Using the effective radii determined by Crnojević et al. (2014) via deep CFHT/PAndAs imaging, this corresponds to 1.3 and 2.3 effective radii from each galaxy center.

The combined ACS exposure times were $82280 \mathrm{~s}$ (23 hours) for NGC 147 and 62598s (17 hours) for NGC 185. Individual exposures were well dithered to fill in the gap between the two ACS chips and improve the point-spread function (PSF). Short 30-second exposures were taken as part of the program, but were not included in our reduced image stacks as bright stars are not the focus of this paper (individual long exposures saturate brighter than $V \sim 20$ ). The ACS field centers and total exposure times are listed in Table 1. The location of all fields, including parallel WFC3 and archival WFPC2 pointings, are shown in Figure 1.

Our ACS images were processed through the HST archive on-the-fly reprocessing system using the most upto-date calibration frames. The images were corrected for CCD charge transfer efficiency losses following the prescription in Anderson \& Bedin (2010). We then coadded the images into a single image per field per filter using the MULTIDRIZZLE software (Fruchter \& Sosey 2009), which also corrects for geometric distortion and removes bad pixels and cosmic rays. Due to the multiply 

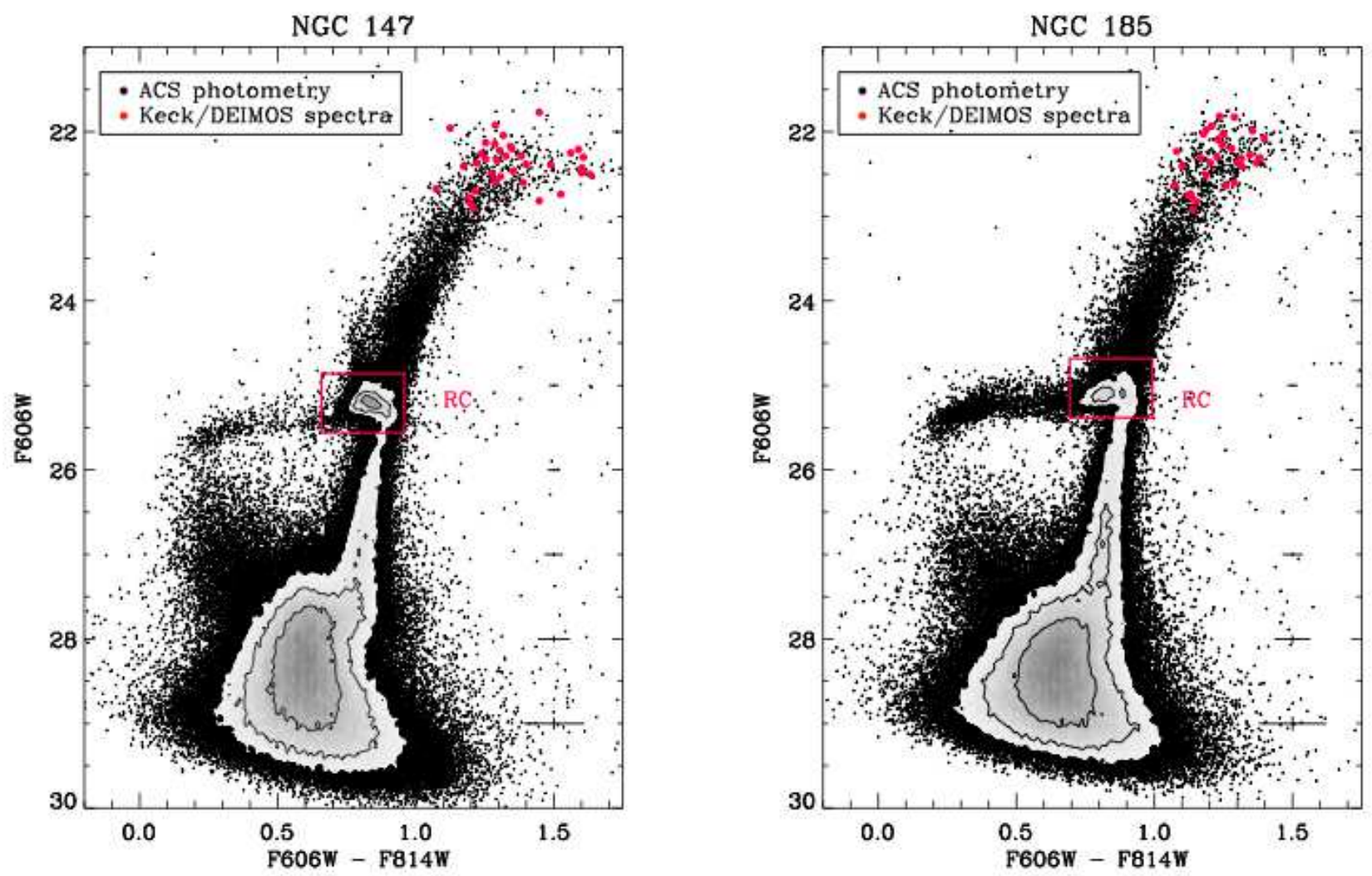

FIG. 2.- Color-magnitude diagrams based on HST/ACS data for NGC 147 (left) and NGC 185 (right). One sigma error bars are indicated to the right of each CMD. Red symbols show the position of member stars in each ACS field with Keck/DEIMOS spectroscopic metallicity measurements. While initially these two CMDs appear similar, closer inspection shows differences in the Red Clump (RC), horizontal branch and main sequence turnoff regions.

dithered images, the ACS images were resampled to a final pixel size of $0.04^{\prime \prime}$ ( 0.8 times the native plate scale).

PSF-fitting photometry was performed using the DAOPHOT/ALLSTAR II package (Stetson 1994). To create the PSF model we chose $>400$ stars with good spatial coverage in each filter for each galaxy. Nearby neighbors were subtracted from around the PSF stars and the cleaned stars were then used to make the final PSFs. To find faint stars, we performed a single iteration of fitting the known stars, subtracting them from the images and using this image to search for faint companions. We then re-performed the PSF fitting photometry on the entire original image using the updated catalog. Stars were selected if they were found in both filters within a matching radius of 0.5 pixels and passed the following criteria: $\sigma_{F 606, F 814}<0.15 \mathrm{mag}, \chi^{2}<3$ and abs(sharpness) $<0.65$. The number of stars in each field is listed in Table 1. Instrumental magnitudes were converted into the ACS VEGAmag system using the procedure outlined by Sirianni et al. (2005), but with updated ACS zeropoints (Bohlin 2007).

Artificial star tests were performed by adding a total of a half million artificial stars in each image stack. Artificial stars were spread evenly across the full frame and spaced such that they were separated from each other by at least twice the PSF radius. PSF fitting photometry was then performed on the entire frames, measuring both the real and artificial stars, using the same steps as above. Artificial stars were recovered if they passed the same photometric criteria above. The $50 \%$ completeness levels are $\mathrm{F} 606 \mathrm{~W}=29.05$ and $28.95 \mathrm{mag}$, and $\mathrm{F} 814 \mathrm{~W}=$ 28.35 and $28.25 \mathrm{mag}$ for NGC 147 and NGC 185, respectively.

Contamination from either foreground Milky Way stars or stars associated with the M31 halo is expected to be minimal in our ACS fields. Using the Besançon model of the Milky Way (Robin et al. 2003), we calculate there are $\sim 40$ Milky Way stars contained within each ACS field-of-view in the magnitude and color range $\mathrm{F} 814 \mathrm{~W}=22-28$ and $(\mathrm{F} 606 \mathrm{~W}-\mathrm{F} 814 \mathrm{~W})<1.5$. This is negligible compared to the hundreds of thousands of stars detected in each dE galaxy (see Table 1). For the model fitting in $\S 3.3$, we do account for Milky Way foreground contamination using the empirical models of de Jong et al. (2010). The contamination from M 31 stars at a projected radius of $7^{\circ}$ or $100 \mathrm{kpc}$ is less than that from the Milky Way foregrounds and we do not account for this in the models.

The resulting HST ACS CMDs are shown for NGC 147 and NGC 185 in Figure 2. We plot one sigma errors as a function of magnitude. These represent the deepest CMDs for any $\mathrm{dE}$ galaxy and are the first observations of dE galaxy stars below the main sequence turnoff.

\subsection{Distances and Spectroscopic Metallicities}

Given the extremely small photometric errors in our data, we chose to re-determine the distance of NGC 147 and NGC 185 using the $H S T /$ ACS data itself via the Tip of the Red Giant Branch (TRGB) method. The TRGB acts as a discontinuity in the stellar luminosity function, the position of which is easily measured. We measure 

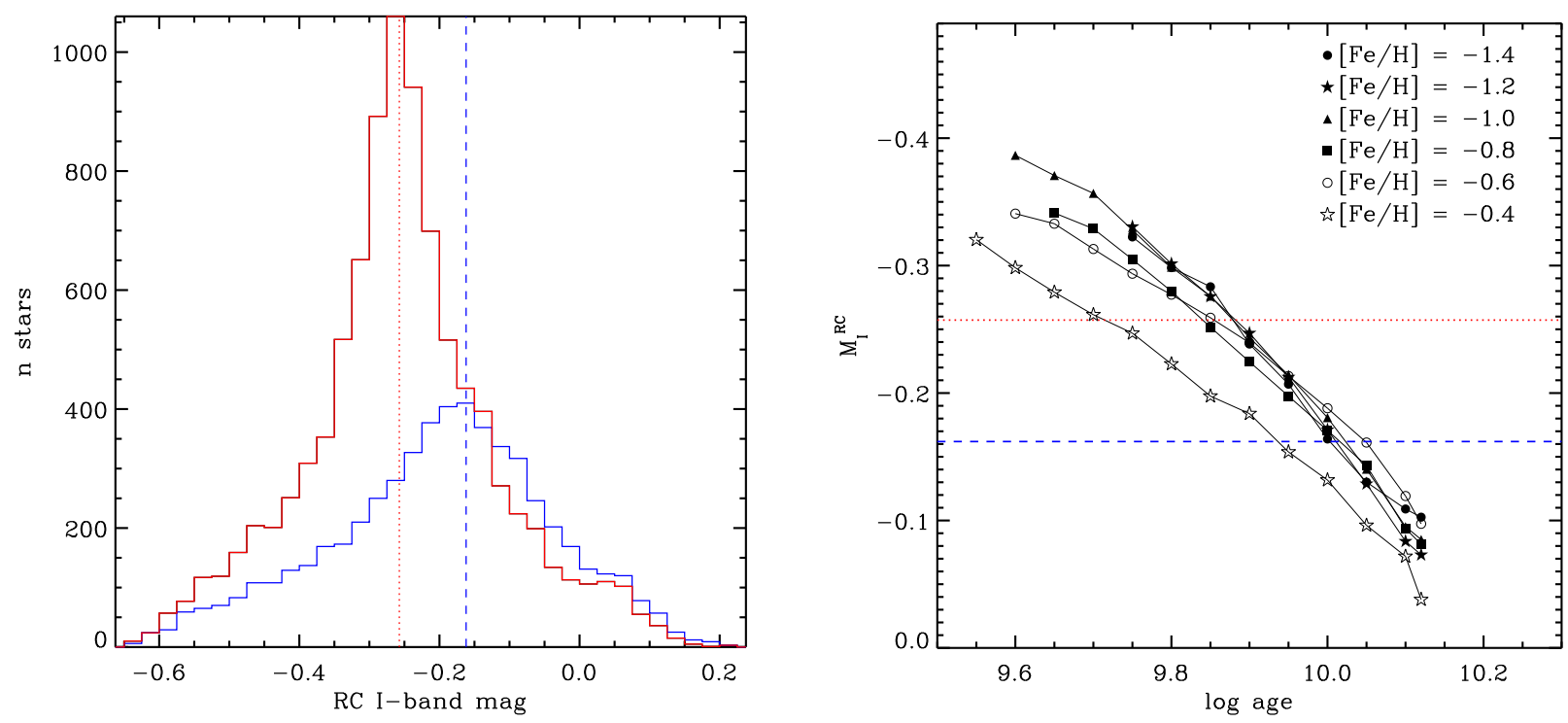

FIG. 3.- As a first comparison of the SFHs, we compare the observed distribution of stars near the Red Clump with single isochrone models. The red clump region of the CMD is shown inside the red box of Figure 2 The peak of this distribution in absolute magnitude (left) occurs 0.1 magnitudes brighter for NGC 147 (red) as compared to NGC 185 (blue). Comparing to isochrone predictions (right) for a range of metallities $([\mathrm{Fe} / \mathrm{H}]$ between -0.4 to -1.4$)$, this implies a younger mean age for NGC 147 .

the TRGB using the software developed by one of us (R.P.v.d.M.) and detailed in Cioni et al. (2000). The distance modulus for NGC 147 is $(m-M)_{0}=24.30 \pm 0.05$ $(724 \pm 27 \mathrm{kpc})$ and NGC 185 is $(m-M)_{0}=24.02 \pm 0.08$ $(636 \pm 26 \mathrm{kpc})$. These are slightly further, but well within the 1- $\sigma$ errors, of those determined by Conn et al. (2012). We assume a distance to M 31 of $779 \mathrm{kpc}$ from Conn et al. (2012). This places NGC 147 and NGC 185 at distances 165 and $210 \mathrm{kpc}$ away from their parent galaxy M 31, respectively. We adopt extinction values for NGC 147 and NGC 185 from Schlegel et al. (1998) of $\mathrm{E}(\mathrm{B}-\mathrm{V})=0.161$ and 0.195 .

Spectroscopic metallicities for stars in the ACS fields were obtained as part of a larger Keck/DEIMOS study of Local Group dEs (Geha et al. 2010; Vargas et al. 2014; Ho et al. 2015). We use these spectroscopic metallicities to guide our single stellar population analysis in $\S 3.1$ The Keck sample includes 37 member stars within our ACS field-of-view in NGC 147, and 30 member stars in the NGC 185 ACS field. Members are identified as having colors, magnitudes and radial velocities consistent with each galaxy. Spectroscopic metallicities $([\mathrm{Fe} / \mathrm{H}])$ of individual RGB stars were determined using the spectral synthesis method described in Vargas et al. (2014). The mean metallicity within each ACS field is $<[\mathrm{Fe} / \mathrm{H}]>=$ $-0.5 \pm 0.1$ for $\mathrm{NGC} 147$ and $<[\mathrm{Fe} / \mathrm{H}]>=-1.0 \pm 0.1$ for NGC 185, comparable to the means of each full sample. While the error on the mean is small, there is significant internal scatter of $0.5 \mathrm{dex}$ in each field. While the analysis below assumes solar alpha-abundance ratios, we note that the measured alpha-abundances are slightly enhanced at $[\alpha / \mathrm{Fe}]=0.3 \pm 0.1$ in NGC 147 and $[\alpha / \mathrm{Fe}]=0.1 \pm 0.1$ in NGC185 (Vargas et al. 2014).

\section{RESULTS}

In Figure 2 the $H S T /$ ACS CMDs for NGC 147 and NGC 185 appear similar at first glance, yet clear differences emerge on closer inspection. Focusing first on the position of stars which have evolved off the main sequence, NGC 147 has a strong Red Clump (RC), while stars in the same evolutionary state in NGC 185 are instead distributed equally between the $\mathrm{RC}$ and a bluer horizontal branch, consistent with a lower average metallicity in NGC 185. Also seen in the CMDs of Figure 2 is the larger number of stars bluer and brighter than the main sequence turnoff in NGC 147 as compared to NGC 185. While some fraction of these stars are most likely blue straggler stars (Santana et al.2013), this does suggest the presence of a younger population in NGC 147 as compared to NGC 185. Based on this visual inspection alone, we conclude that the star formation histories of these two dEs are different. Below, we quantify this statement via both simple comparison to single population isochrones $(\oint 3.1)$ and CMD fitting to determine the full star formation histories $(\oint 3.3)$.

\subsection{Simple Constraints on the Distribution of Stellar Ages}

Before modeling the inferred star formation histories from the full CMDs, we first compare the data to single stellar population isochrones to build a basic understanding of these data. We use the Padova isochrones (Girardi et al. 2002, 2010) generated in the HST ACS filters with solar abundance ratios.

We focus on the relative ages of NGC 147 and NGC 185 by comparing stars in the Red Clump (RC). The location of the RC is shown in Figure 2, For metallicities more metal-poor than $\sim-0.5$ dex, the F814W (roughly $I$-band) luminosity of the $\mathrm{RC}$ is largely dominated by age effects. In the left panel of Figure 3, we plot the F814W luminosity functions of RC stars in NGC 147 (red) and NGC 185 (blue). NGC 147's median RC magnitude is marked by the red dotted vertical line, while the blue dashed line marks NGC 185's RC magnitude. In the right panel of Figure 3 we compare the median RC luminosity to the values predicted for single age/metallicity stellar populations. These values were determined using the same RC boxes as the observations.

Spectroscopic abundances for NGC 147 and NGC 185 

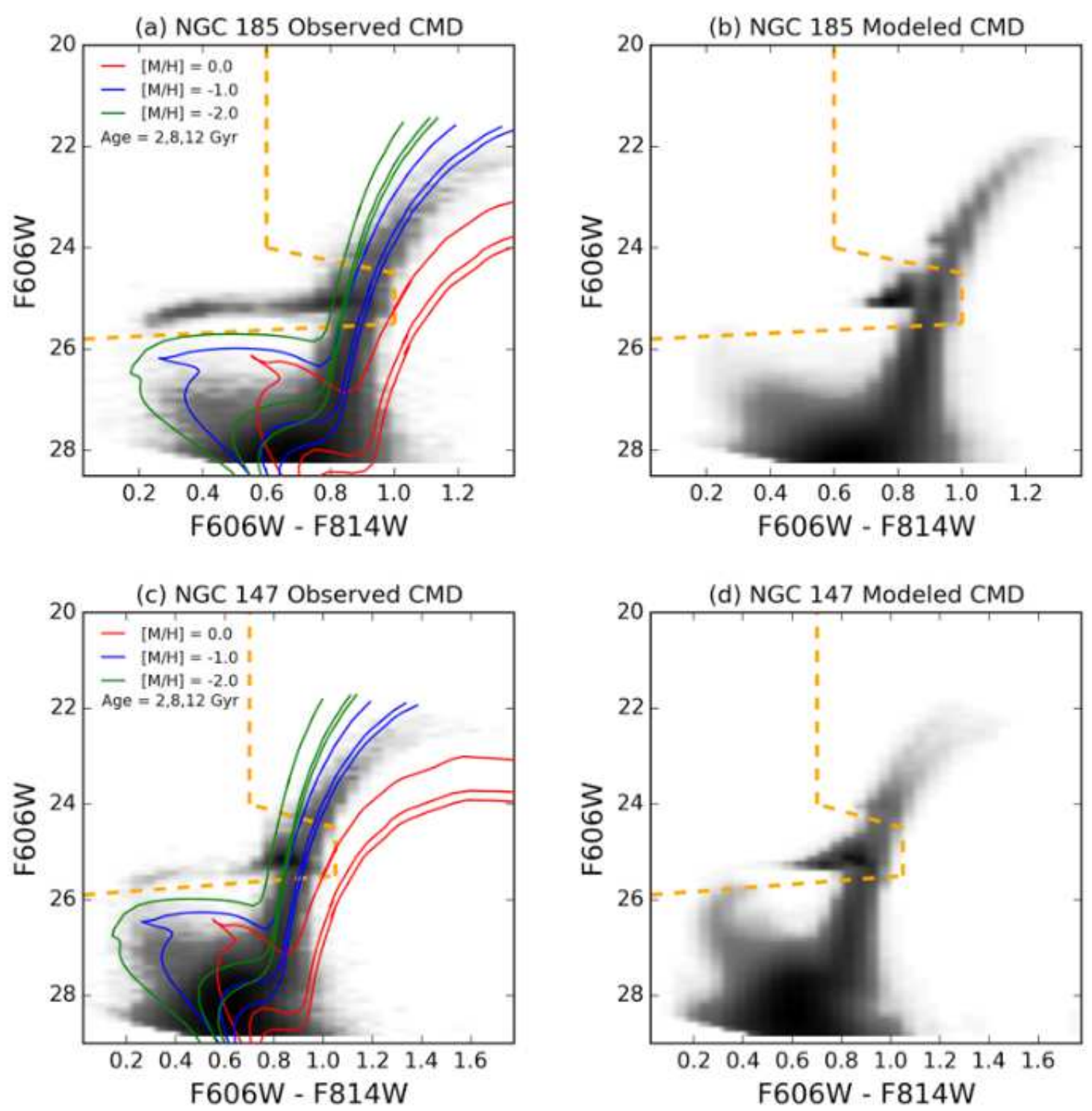

FIG. 4.- Left panels: The observed Hess diagrams for NGC 185 (top) and NGC 147 (bottom). Overplotted are Padova isochrones with $[\mathrm{Fe} / \mathrm{H}]=-2$ (green), -1 (blue) and $0.0 \mathrm{dex}$ (red) for ages of 2, 8 and $12 \mathrm{Gyr}$. (Right panels:) The best fitting model star formation histories as determined using MATCH. The region above and to the left of the orange boundaries are not used in the CMD fitting.

are $[\mathrm{Fe} / \mathrm{H}]=-0.5$ and -1.0 , respectively $(\S 2.2)$, thus we can use the $\mathrm{RC}$ to determine the relative ages of the two galaxies. The dominant RC population in NGC 147 has an age of $\sim 7 \mathrm{Gyr}$, while NGC 185's is $\sim 11$ Gyr old. This makes NGC 185 about 4 Gyr older. This is in agreement with the full SFHs discussed in $\S 3.3$ and serves an as independent check of those result, as the full SFHs are determined without including this region of the CMD.

In the left panels of Figure 4, we overplot onto the CMD Hess diagrams representative isochrones spanning a wide range of metallicities $([\mathrm{Fe} / \mathrm{H}]=0,-1$ and -2$)$. For the best fitting set of isochrones which fits the red giant branch (blue lines for both dEs), the bulk of main sequence turn-off stars lie between the 2 and 8 Gyr tracks for NGC 147, while these stars primarily lie between the 8 to 12 Gyr tracks for NGC 185 . Finally, if all of the stars bluer and brighter than the main sequence turnoff are interpreted as young stars (some fraction must be older blue straggler stars), than this population is likely not younger then $\sim 2$ Gyrs. We explore this in more detail below.

\subsection{Measuring Full Star Formation Histories}

We measure the full SFHs of both galaxies using the CMD fitting package MATCH (Dolphin 2002). MATCH constructs a set of synthetic simple stellar populations which are linearly combined and added to a model foreground population from the empirical model in de Jong et al. (2010) to form a composite synthetic CMD. This CMD is then convolved with results from artificial star tests. The models and observed CMDs are compared using a Poisson likelihood statistic. The SFH that corresponds to the best matched synthetic $\mathrm{CMD}$ is the most likely $\mathrm{SFH}$ of the observed population. The fitting of these CMDs uses the Padova stellar models (Girardi et al. 2002, 2010) and follows the fitting methodology from Weisz et al. (2014). We assume a Kroupa IMF, solar-scaled isochrones and the 

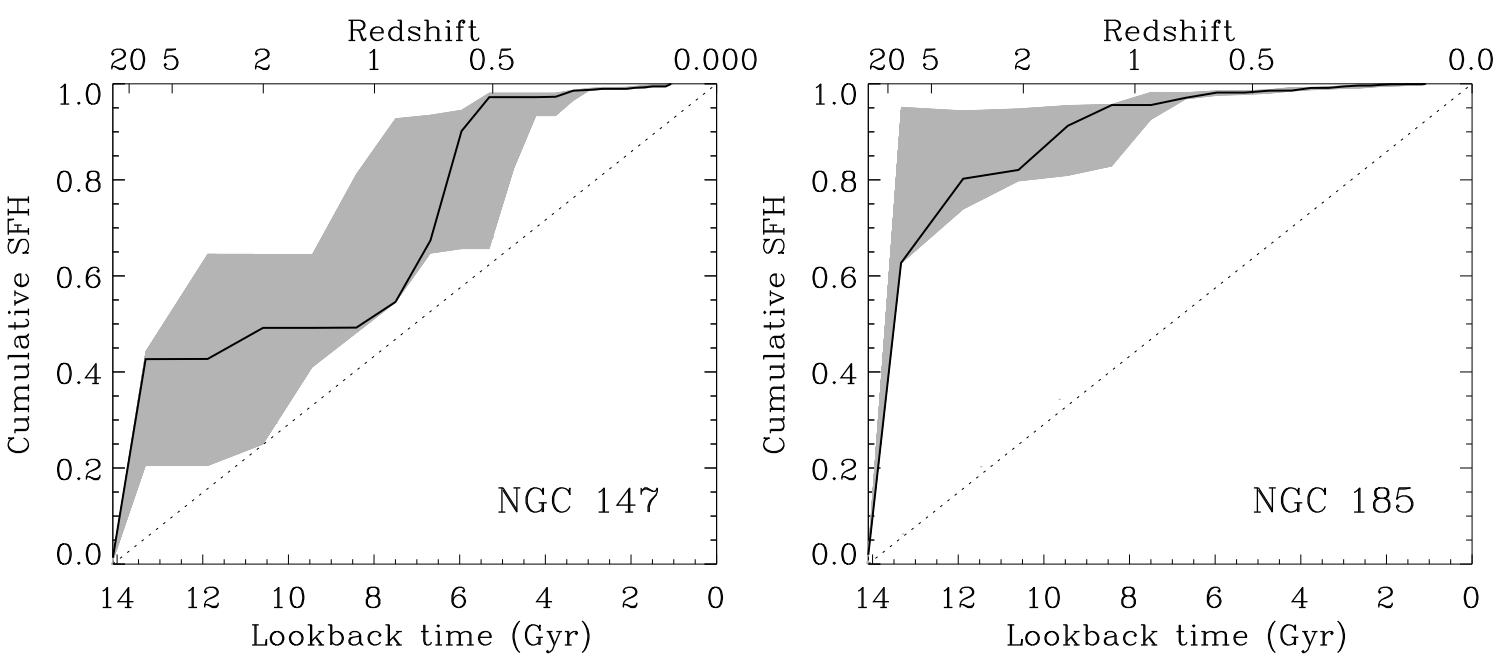

FIG. 5.- Cumulative SFHs for NGC 147 (left) and NGC 185 (right) from the best-fitting SFHs models shown in Figure 4 The shaded envelope reflects the $68 \%$ confidence interval around the best fit SFH. The dotted line indicates a constant star formation rate over the lifetime of the galaxy. While the NGC 185 field is primarily old, the bulk of stars in NGC 147 formed between 5-7 Gyr ago.

distances/reddening stated in $\$ 2.2$ We exclude the red clump and horizontal branch regions from the fit in order to mitigate the contribution of these relatively less certain phases of stellar evolution to the SFH (Aparicio \& Hidalgo 2009). The excluded region is shown above and to the left of the orange dashed lines in Figure 4

Uncertainties in the SFHs reflect the $68 \%$ confidence interval around the best fit SFH due to both random uncertainties (from a finite number of stars on the CMD) and systematic uncertainties (due to uncertain physics in the underlying stellar models). In both our ACS datasets, systematic errors dominate over random. We refer the reader to Dolphin (2012) for a full discussion of systematic uncertainties and Dolphin (2013) for a detailed description of random uncertainties in $\mathrm{SFH}$ measurements.

\subsection{Full SFH Results}

The best-fitting MATCH CMD models are compared to the observed Hess diagrams in Figure 4. The cumulative star formation histories from these models and their uncertainties are shown in Figure 5. The main features of the main sequence, main sequence turnoff and upper red giant branches are generally well fit. We note minor discrepancies between the model and data, particularly near the bright end of the youngest main sequence turnoff in NGC 147. This population represents $\sim 1 \%$ of the total stellar mass and may be due to our assumption of solar alpha-abundance ratios or some other unaccounted for systematic. The cumulative SFHs of NGC 147 and NGC 185 required to achieve the overall agreement are significantly different from each other and are consistent with the general conclusion from $\S 3.1$ that NGC 185 hosts a much older stellar population as compared to NGC 147.

In NGC 147, less than half of stars $(40 \%)$ were in place 12.5 Gyrs ago $(z \sim 5)$, with the bulk of the remaining population forming between 5 to 7 Gyrs. Star formation in this ACS field appears to have been quenched roughly 5 Gyrs ago. This agrees with SFHs determined from shal- lower $H S T$ /WFPC2 data from the more central regions of NGC 147 (Weisz et al. 2014). There are no metallicity gradients observed in this galaxy (Vargas et al. 2014; Crnojević et al. 2014), suggesting that the inferred SFH in this ACS field may be representative of the galaxy as a whole. Crnojević et al. (2014) has recently uncovered isophotal twisting at large radius due to the emergence of symmetric tidal tails in NGC 147. Our ACS field is well inside the radius at which these tidal effects are visibly observed.

The NGC 185 field, in contrast, formed $70 \%$ of stars prior to 12.5 Gyrs ago, with the majority of the remaining population forming between 8 to 10 Gyrs ago. Given NGC 185's lower measured spectroscopic metallicity, an older age for NGC 185 relative to NGC 147 is in agreement with expectations from simple models of chemical evolution (e.g., Tinsley 1979). We note that the spectroscopic metallicities are not used as part of the fitting method. The presence of the smaller intermediate age (8-10 Gyr) population is independently confirmed by AGB carbon stars present in the ACS field (Battinelli \& Demers 2004). A radial gradient in both carbon stars and overall stellar metallicity is observed in NGC 185 (Vargas et al. 2014; Crnojević et al. 2014), which suggests that NGC 185 has experienced little to no radial mixing. The star formation in the ACS field, which lies at 2.3 effective radius from the galaxy center $\left(6.7^{\prime}\right.$ or $\left.1.2 \mathrm{kpc}\right)$, is among the oldest of any Local Group dwarf galaxy (Weisz et al. 2014). This is in contrast to the inner $200 \mathrm{pc}$ of NGC 185 which shows evidence for very recent star formation as young as 100 Myrs ago.

\section{FORMATION HISTORY OF NGC147 VERSUS NGC 185}

Based on the star formation histories above, NGC 147 and NGC 185 built up their present-day stellar mass over very different timescales. A key question is whether these galaxies had different formation histories prior to falling into the M31 environment, or if their early histories were similar, but each fell into the M31 potential at very different times. Below we argue that a different infall time into M31 is the most plausible explanation for these dif- 
ferent SFHs.

NGC147 and NGC 185 lie very close on the sky $\left(58^{\prime}\right)$ and have been interpreted either as a bound galaxy pair or a single group that fell together into M31 (van den Bergh 1998). Kinematic evidence marginally suggests that these are not gravitationally bound (Geha et al. 2010; Watkins et al. 2013). Our star formation histories, combined with the presence of a tidal tail in NGC 147 (and the lack thereof in NGC 185) suggest that these two $\mathrm{dE}$ galaxies are independent of each other and that their current proximity is likely a chance projection. While current data only support this conclusion indirectly, future proper motion measurements can definitively determine whether or not these two systems have different orbital histories.

An outstanding issue in comparing NGC 147 to NGC 185 is the difference in gas and dust content. NGC 185 contains some gas and dust $\left(M_{\text {gas }}=3 \times 10^{5} M_{\text {sun }}\right.$, $M_{\text {dust }}=5 \times 10^{3} M_{\text {sun }}$, De Looze et al. in prep., Marleau et al. (2010)), consistent with recycling from older stars. NGC 147 contains no detected gas nor dust $\left(M_{\text {gas }}<3 \times 10^{3} M_{\text {sun }}\right.$, Young \& Lo (1997); $M_{\text {dust }}<$ $128 M_{\text {sun }}$, De Looze et al. in prep.). The lack of gas/dust in NGC 147 has been referred to as the 'missing ISM problem' (Sage et al. 1998). We hypothesize that NGC 147 has had stronger interactions with M 31 as compared to NGC 185 due to an orbit which bring it deeper into the M31 potential, stripping gas/dust and creating its observed tidal tails. On the other hand, we suggest that the orbit of NGC 185 has a larger comparable orbital pericenter, allowing it to maintain a small gas reservoir at its center. We interpret the very recent star formation confined to the central 200 pc of NGC 185 as having formed from this recycled material (Goncalves et al. 2012), which was perhaps triggered during a recent passage through pericenter.

From Figure [5, we concluded that neither the NGC 147 nor NGC 185 field have had a significant star formation event in several gigayears (we do not consider the above $100 \mathrm{Myr}$ central burst in NGC 185 significant). If we assume that star formation is instantaneously quenched once a satellite passes the M31 virial radius, we conclude infall times of 5 and 8 Gyrs for NGC 147 and NGC 185 respectively. We define 'quenched' as having $90 \%$ of stars in place, choosing this value (rather than $100 \%$ ) due to ambiguity with blue straggler stars which are not accounted for in the models. If there is a delay time between infall and star formation quenching (e.g., Wetzel et al. 2013), then these times could be much earlier. Because the majority of stellar mass in NGC 185 was in place earlier than NGC 147, we conclude an earlier infall epoch for this galaxy regardless of the quenching timescale.

Finally, we compare the cumulative SFHs of NGC 147 and NGC 185 to other dwarf galaxy satellites around the Milky Way and M31 from Weisz et al. (2014). NGC 147 is typical of other luminous Local Group satellites, with evidence for a mixture of both old (12 Gyr) and more intermediate age stars. On the other hand, our NGC 185 field is more typical of the predominately old populations seen in much lower stellar mass systems such as Sextans and Draco. In these lower mass systems, star formation is assumed to have been suppressed due to reionization. However, NGC 185 has a factor of 100 more stellar mass than these systems and unlikely to be affected to this extent by reionization. Instead this may be further evidence for NGC 185's early infall time into the M31 environment.

\section{CONCLUSIONS}

We present deep HST ACS photometry of the M31 dE satellite galaxies NGC 147 and NGC 185. These data are the first to reach below the main sequence turnoff in a $\mathrm{dE}$ galaxy and allow us to unambiguously determine their star formation histories. We find a much older population in the dE galaxy NGC 185 as compared to NGC 147: while $70 \%$ of stars were already formed in NGC 185 before 12.5 Gyrs, only $40 \%$ of the present day stellar population had formed in NGC 147. We broadly interpret these results to imply an earlier infall time into the M31 environment for NGC 185 versus NGC 147. We further suggest that the orbit of NGC 185 has a larger pericenter as compared to NGC 147, allowing it to preserve radial age/metallicity gradients and maintain a small central reservoir of recycled gas. These conclusions could be confirmed via future proper motion measurements of these two systems to determine their 3D orbits around M31.

Support for this work was provided by NASA through grant number HST GO-11724 and HST-GO-10794 from the Space Telescope Science Institute, which is operated by AURA, Inc., under NASA contract NAS5-26555. We thank Ana Bonaca, Jeremy Bradford and Ilse de Looze for comments on the draft. MG acknowledges support from NSF grant AST-0908752. Support for DRW is provided by NASA through Hubble Fellowship grant HSTHF-51331.01 awarded by the Space Telescope Science Institute, which is operated by the Association of Universities for Research in Astronomy, Inc., under NASA contract NAS 5-26555. PG acknowledges support from NSF grant AST-1412648.

\section{REFERENCES}

Anderson, J. \& Bedin, L. R. 2010, PASP, 122, 1035

Aparicio, A. \& Hidalgo, S. L. 2009, AJ, 138, 558

Battinelli, P. \& Demers, S. 2004, A\&A, 418, 33

Bender, R., Paquet, A., \& Nieto, J.-L. 1991, A\&A, 246, 349

Binggeli, B., Sandage, A., \& Tammann, G. A. 1988, ARA\&A, 26, 509

Bohlin, R. C. 2007, Photometric Calibration of the ACS CCD Cameras, Tech. rep.

Butler, D. J. \& Martínez-Delgado, D. 2005, AJ, 129, 2217

Choi, P. I., Guhathakurta, P., \& Johnston, K. V. 2002, AJ, 124, 310
Cioni, M.-R. L., van der Marel, R. P., Loup, C., \& Habing, H. J. 2000, A\&A, 359, 601

Conn, A. R., Ibata, R. A., Lewis, G. F., Parker, Q. A., Zucker, D. B., Martin, N. F., McConnachie, A. W., Irwin, M. J., Tanvir, N., Fardal, M. A., Ferguson, A. M. N., Chapman, S. C., \& Valls-Gabaud, D. 2012, ApJ, 758, 11

Crnojević, D., Ferguson, A. M. N., Irwin, M. J., McConnachie, A. W., Bernard, E. J., Fardal, M. A., Ibata, R. A., Lewis, G. F., Martin, N. F., Navarro, J. F., Noël, N. E. D., \& Pasetto, S. 2014, MNRAS, 445, 3862

Davidge, T. J. 2005, AJ, 130, 2087 
TABLE 1

HST ACS OBSERVATIONS

\begin{tabular}{|c|c|c|c|c|}
\hline Row & Quantity & Units & NGC 147 & NGC 185 \\
\hline (1) & RA & $h: m: s$ & $00: 33: 12.1$ & $00: 38: 58.0$ \\
\hline (2) & DEC & ${ }^{\circ}:{ }^{\prime}:{ }^{\prime \prime}$ & $+48: 30: 31$ & $+48: 20: 15$ \\
\hline (3) & $\mathrm{E}(\mathrm{B}-\mathrm{V})$ & mag & 0.161 & 0.195 \\
\hline (4) & $(\mathrm{M}-\mathrm{m})_{0}$ & & $24.30 \pm 0.05$ & $24.02 \pm 0.08$ \\
\hline$(5)$ & Dist & $\mathrm{kpc}$ & $724 \pm 27$ & $636 \pm 26$ \\
\hline (6) & $M_{V, 0}$ & mag & $-16.5 \pm 0.04$ & $-15.5 \pm 0.04$ \\
\hline (7) & $r_{\mathrm{eff}}$ & 1 & $6.7 \pm 0.09$ & $2.94 \pm 0.04$ \\
\hline (8) & $r_{\text {eff }}$ & $\mathrm{kpc}$ & $1.41 \pm 0.02$ & $0.53 \pm 0.01$ \\
\hline (9) & {$[\mathrm{Fe} / \mathrm{H}]$} & dex & $-0.5 \pm 0.1$ & $-0.9 \pm 0.1$ \\
\hline$(10)$ & $\mathrm{RA}_{\mathrm{HST} / \mathrm{ACS}}$ & $\mathrm{h}: \mathrm{m}: \mathrm{s}$ & $00: 32: 51.0$ & $00: 39: 10.5$ \\
\hline (11) & $\mathrm{DEC}_{\mathrm{HST} / \mathrm{ACS}}$ & $\circ: '{ }^{\circ}: " 1$ & $+48: 22: 39.8$ & $+48: 26: 35.9$ \\
\hline$(12)$ & $\mathrm{t}_{\mathrm{exp}, \mathrm{F} 606 \mathrm{~W}}$ & hrs & 10.65 & 12.2 \\
\hline (13) & $\mathrm{t}_{\mathrm{exp}, \mathrm{F} 814 \mathrm{~W}}$ & hrs & 7.54 & 9.84 \\
\hline (14) & $N_{\text {stars }}$ & & 277,949 & 197,634 \\
\hline
\end{tabular}

Note. - Columns (1)-(3) are taken from NED for the galaxy center. Columns (3) from Schlegel et al. (1998); column (4)-(5) are determined from our photometry as discussed in $\S 2.2$ Columns (6)-(8) are from Crnoiević et al. (2014); column (9) from Vargas et al. (2014).

The last five columns are specific to the HST/ACS data presented

here.

de Jong, J. T. A., Yanny, B., Rix, H.-W., Dolphin, A. E., Martin, N. F., \& Beers, T. C. 2010, ApJ, 714, 663

Dolphin, A. E. 2002, MNRAS, 332, 91

-. 2012, ApJ, 751, 60

-. 2013, ApJ, 775, 76

Ferguson, H. C. \& Sandage, A. 1991, AJ, 101, 765

Fruchter, A. S. \& Sosey, M. 2009, The MultiDrizzle Handbook, Version 3, Baltimore, STScI

Geha, M., Blanton, M. R., Yan, R., \& Tinker, J. L. 2012, ApJ, 757,85

Geha, M., Guhathakurta, P., \& van der Marel, R. P. 2002, AJ, 124,3073

-. 2003, AJ, 126, 1794

Geha, M., van der Marel, R. P., Guhathakurta, P., Gilbert, K. M., Kalirai, J., \& Kirby, E. N. 2010, ApJ, 711, 361

Girardi, L., Bertelli, G., Bressan, A., Chiosi, C., Groenewegen, M. A. T., Marigo, P., Salasnich, B., \& Weiss, A. 2002, A\&A, 391,195

Girardi, L., Williams, B. F., Gilbert, K. M., Rosenfield, P., Dalcanton, J. J., Marigo, P., Boyer, M. L., Dolphin, A., Weisz, D. R., Melbourne, J., Olsen, K. A. G., Seth, A. C., \& Skillman E. 2010, ApJ, 724, 1030

Gonçalves, D. R., Magrini, L., Martins, L. P., Teodorescu, A. M., \& Quireza, C. 2012, MNRAS, 419, 854

Ho, N., Geha, M., Tollerud, E. J., Zinn, R., Guhathakurta, P., \& Vargas, L. C. 2015, ApJ, 798, 77

Janz, J., Laurikainen, E., Lisker, T., Salo, H., Peletier, R. F., Niemi, S.-M., den Brok, M., Toloba, E., Falcón-Barroso, J., Boselli, A., \& Hensler, G. 2012, ApJ, 745, L24

Lisker, T., Janz, J., Hensler, G., Kim, S., Rey, S., Weinmann, S., Mastropietro, C., Hielscher, O., Paudel, S., \& Kotulla, R. 2009, ApJ, 706, L124

Lisker, T., Weinmann, S. M., Janz, J., \& Meyer, H. T. 2013, MNRAS, 432, 1162

Marleau, F. R., Noriega-Crespo, A., \& Misselt, K. A. 2010, ApJ, 713,992

Mastropietro, C., Moore, B., Mayer, L., Debattista, V. P., Piffaretti, R., \& Stadel, J. 2005, MNRAS, 364, 607

Mayer, L. 2010, Advances in Astronomy, 2010

Michielsen, D., Boselli, A., Conselice, C. J., Toloba, E., Whiley, I. M., Aragón-Salamanca, A., Balcells, M., Cardiel, N., Cenarro, A. J., Gorgas, J., Peletier, R. F., \& Vazdekis, A. 2008, MNRAS, 385,1374
Monachesi, A., Trager, S. C., Lauer, T. R., Hidalgo, S. L., Freedman, W., Dressler, A., Grillmair, C., \& Mighell, K. J. 2012, ApJ, 745, 97

Moore, B., Lake, G., \& Katz, N. 1998, ApJ, 495, 139

Robin, A. C., Reylé, C., Derrière, S., \& Picaud, S. 2003, A\&A, 409, 523

Ryś, A., Falcón-Barroso, J., \& van de Ven, G. 2013, MNRAS, 428,2980

Sage, L. J., Welch, G. A., \& Mitchell, G. F. 1998, ApJ, 507, 726

Saha, A. \& Hoessel, J. G. 1990, AJ, 99, 97

Santana, F. A., Muñoz, R. R., Geha, M., Côté, P., Stetson, P., Simon, J. D., \& Djorgovski, S. G. 2013, ApJ, 774, 106

Schlegel, D. J., Finkbeiner, D. P., \& Davis, M. 1998, ApJ, 500, 525

Sirianni, M., Jee, M. J., Benítez, N., Blakeslee, J. P., Martel, A. R., Meurer, G., Clampin, M., De Marchi, G., Ford, H. C., Gilliland, R., Hartig, G. F., Illingworth, G. D., Mack, J., \& McCann, W. J. 2005, PASP, 117, 1049

Sohn, Y., Kang, A., Rhee, J., Shin, M., Chun, M., \& Kim, H. 2006, A\&A, 445, 69

Stetson, P. B. 1994, PASP, 106, 250

Tinsley, B. M. 1979, ApJ, 229, 1046

Toloba, E., Guhathakurta, P., Peletier, R. F., Boselli, A., Lisker T., Falcón-Barroso, J., Simon, J. D., van de Ven, G., Paudel, S., Emsellem, E., Janz, J., den Brok, M., Gorgas, J., Hensler, G., Laurikainen, E., Niemi, S.-M., Ryś, A., \& Salo, H. 2014, ApJS, 215, 17

van den Bergh, S. 1998, AJ, 116, 1688

van Zee, L., Barton, E. J., \& Skillman, E. D. 2004, AJ, 128, 2797

Vargas, L. C., Geha, M. C., \& Tollerud, E. J. 2014, ApJ, 790, 73

Veljanoski, J., Ferguson, A. M. N., Huxor, A. P., Mackey, A. D., Fishlock, C. K., Irwin, M. J., Tanvir, N., Chapman, S. C.,

Ibata, R. A., Lewis, G. F., \& McConnachie, A. 2013, MNRAS, 435,3654

Watkins, L. L., Evans, N. W., \& van de Ven, G. 2013, MNRAS, 430, 971

Weisz, D. R., Dolphin, A. E., Skillman, E. D., Holtzman, J., Gilbert, K. M., Dalcanton, J. J., \& Williams, B. F. 2014, ApJ, 789,147

Wetzel, A. R., Tinker, J. L., Conroy, C., \& van den Bosch, F. C. 2013, MNRAS, 432, 336

Yang, S. \& Sarajedini, A. 2010, ApJ, 708, 293

Young, L. M. \& Lo, K. Y. 1997, ApJ, 476, 127 